\title{
Viability, Yield and Expansion Capability of Feline MSCs Obtained from Subcutaneous and Reproductive Organ Adipose Depots
}

\section{Amy Wysong}

Kansas City University of Medicine and Biosciences

Priscilla Ortiz

Innova Celulas Madre

Douglas Bittel

Kansas City University of Medicine and Biosciences

Lindsey Ott

Likarda, LLC

\section{Francis Karanu}

Likarda, LLC

\section{Michael Filla}

Kansas City University of Medicine and Biosciences

Lisa Stehno-Bittel ( $\square$ lbittel@kumc.edu )

University of Kansas Medical Center https://orcid.org/0000-0002-8879-6124

\section{Research article}

Keywords: Feline, MSC, Multipotent stem cells, Expansion, Gata6, Sox2, Sox17

Posted Date: August 31st, 2020

DOl: https://doi.org/10.21203/rs.3.rs-51093/v1

License: (c) (i) This work is licensed under a Creative Commons Attribution 4.0 International License. Read Full License

Version of Record: A version of this preprint was published at BMC Veterinary Research on July 15th, 2021. See the published version at https://doi.org/10.1186/s12917-021-02948-0. 


\title{
Viability, yield and expansion capability of feline MSCs obtained from subcutaneous and reproductive organ adipose depots
}

\author{
Amy Wysong ${ }^{1}$, Priscilla Ortiz ${ }^{2}$, Douglas Bittel ${ }^{1}$, Lindsey Ott ${ }^{3}$, Francis Karanu ${ }^{3}$, Michael Filla ${ }^{1}$, Lisa
} Stehno-Bittel ${ }^{3,4}$

1 College of Biology, Kansas City University, 1750 Independence Ave, Kansas City MO, USA 2 Innova Celulas Madre, San Pedro, San Jose, Costa Rica

3 Likarda LLC, 10330 Hickman Mills Drive, Kansas City MO, USA

4 Department of Rehabilitation Science, University of Kansas Medical Center, 3901 Rainbow Blvd, Kansas City, KS 66160

Correspondence:

Lisa Stehno-Bittel, PhD

Ibittel@kumc.edu 


\section{ABSTRACT}

Background: The source of multipotent stromal cells (MSC) can have a significant influence on the health and expansion capacity of the cells. As the applications for allogeneic MSCs in the treatment of feline diseases increase, the location of the initial donor tissue must be analyzed. To date, comparisons have only been made between feline MSCs collected from bone marrow or abdominal fat. This is the first report to compare cells obtained from different adipose depots in the cat. The adipose tissue was collected from 32 healthy cats undergoing spaying (fat around the ovaries and uterine horn) or subcutaneous fat collected during surgical procedures.

Results: The total tissue yield from the subcutaneous fat was significantly greater than could be obtained from around the reproductive organs, leading to 3 times more total MSCs. However, the density of MSCs obtained from reproductive fat was significantly higher than from subcutaneous fat. In addition, the viability of the MSCs from the reproductive fat was significantly higher than the subcutaneous fat. When sufficient tissue was collected, it was digested either mechanically or enzymatically. Mechanical digestion further decreased the viability and yield of MSCs from subcutaneous fat compared to enzymatic digestion. Biomarkers of stem cell expansion and function were detected using qPCR. Gata6 was detected in all samples similarly regardless of site of harvest or method of digestion. Sox2 and Sox17 were also detected in all samples with higher quantities found in the enzymatically digested subcutaneous fat. Control genes of Gata4 and Pdx1 were detected in very low levels. Negative controls showed no detection prior to 50 cycles. During the first passage there was no statistically significant difference in doubling times or viability. But at P2, MSCs from the enzymatically-digested reproductive fat had the lowest doubling time and a trend towards the highest viability. 
Conclusion: Feline reproductive adipose tissue is a reasonable source of MSCs for eventual therapeutic applications with superior cell density, viability and expansion.

\section{KEYWORDS}

Feline, MSC, Multipotent stem cells, Expansion, Gata6, Sox2, Sox17 


\section{BACKGROUND}

Multipotent stromal cells (MSC) are increasingly popular in regenerative medicine because of the ease of access and isolation, ability to self-renew and multipotent potential [1]. These adult stem cells have the ability to differentiate into cells of the mesodermal lineage such as adipocytes and osteoblasts, but they also have transdifferentiation properties allowing them to be driven to other cell types such as neurons or myocardial cells [2, 3]. Reports have described the therapeutic application of MSCs to treat various conditions such as heart failure, inflammatory diseases, musculoskeletal trauma, diabetes and kidney disease [4, 5]. Additionally, MSCs have successfully been used for treating inflammatory conditions such as osteoarthritis or graft versus host disease [6].

Beyond humans, there are numerous clinical applications for MSCs in the veterinary field, including the treatment of feline diseases such as gingivostomatitis, inflammatory bowel disease, allergic asthma and chronic kidney disease [7-10]. Research on MSC-based treatment for feline ailments is expanding our understanding of the potential of stem cells, along with the challenges associated with feline cells.

MSCs can be harvested from a variety of tissues including adipose tissue, bone marrow, peripheral blood, and umbilical cord [11, 12]. Cells from diverse sites have unique properties including basic transcriptome differences and variations in in vitro or in vivo differentiation [13]. Of the multiple sites from which to obtain MSCs, adipose tissue is one of the most commonly used and widely studied. Historically, adipose tissue was considered a simple site of energy 
storage, but this is no longer the case. Adipose tissue is a functionally dynamic organ that is involved in energy homeostasis and the secretion of several hormones [14].

The abdominal cavity includes several types of adipose tissue, such as subcutaneous, intramuscular and visceral. Subcutaneous adipose tissue lies underneath the skin and is composed of adipocytes that are organized in lobules, separated by connective tissue. Subcutaneous adipose tissue makes up about $80 \%$ of all body fat [15]. In contrast, visceral adipose tissue is interspersed among the organs and composed of several different depots including mesenteric, perirenal, and fat surrounding the reproductive organs. The MSC characteristics can vary depending on the site of tissue harvest [16]. Reports demonstrate differences in MSC characteristics when comparing subcutaneous and visceral adipose tissue surrounding female reproductive tissue obtained during Caesarean section $[17,18]$. Ritter et al concluded that human visceral fat associated with the reproductive organs had a higher potential to differentiate into adipogenic and osteogenic cells and secreted higher levels of inflammatory cytokines, emphasizing the importance of the site of tissue harvest [17].

While studies have compared feline MSCs obtained from either bone marrow or fat tissue [19], no study has compared the different abdominal depots of fat for MSC retrieval in cats. The objective of the study was to compare feline adipose tissue from subcutaneous and reproductive organ donor sites to determine which harvest and digestion protocols resulted in the greatest yield, viability and proliferative biomarkers.

\section{RESULTS}




\section{Donor Characteristics}

All donors in the reproductive fat group were female, as fat was removed during spaying (Table 1). While no statistical analysis could be run on the sex differences, obviously there was a large difference in the sex distribution between the groups. The average age of the donors in the reproductive group was significantly younger than the subcutaneous group again due to the manner of the adipose tissue harvest (Table 1). The average weight of the cats in the two groups was not statistically different (Table 1).

\section{Harvest Yield}

A far greater quantity of tissue was obtained from the subcutaneous procedure with an average of $15.5 \mathrm{~g}$ of tissue taken from each animal (Table 1). Approximately 40 times less tissue by weight could be obtained from the adipose tissue surrounding the reproductive organs. Tissue harvested from the subcutaneous region of each donor was separated into mechanical or enzymatic digestion. Dual digestion protocols could not be tested using the reproductive fat, due to the limited amount of tissue obtained from each donor. Thus, all reproductive fat samples were enzymatically digested.

While more total tissue was obtained from the subcutaneous region, the density of MSCS per gram of tissue was greater for the reproductive fat. Figure 1 illustrates the vast difference in the yield per gram of tissue in the reproductive fat versus the subcutaneous tissue samples. Further, the method of tissue digestion did not alter the results, with mechanical digestion demonstrating the fewest MSCs per tissue weight (not statistically different). Even with the lower 
density of cells, the vastly larger amount of starting material retrieved from the subcutaneous fat samples still resulted in a larger total yield of cells (Table 1 ).

\section{Harvest Viability and Morphology}

Initial viability following enzymatic digestion of reproductive adipose tissue resulted in over $80 \%$ live cells (Figure 2 ). In contrast, there was a statistically significant decrease in viability of cells isolated from the subcutaneous tissue. The viability of the cells obtained from subcutaneous fat was approximately $29 \%$ for the mechanical digestion group, while the value was near $50 \%$ for the enzymatic digestion procedure.

Cells from the two harvest sites adhered well to plastic and formed highly homogenous monolayers. All cells took on the expected fibroblast-like spindle appearance (Figure 3) with the cells from reproductive fat showing the most consistency in shape. There were no noticeable morphological differences between the subcutaneous fat that was enzymatically digested versus mechanical digestion.

\section{Multipotent Biomarker Expression}

Assessment of the extracted RNA demonstrated acceptable quality and concentrations of RNA samples from all groups (Table 2). RNA quality met the anticipated 260/280 ratio of $>1.9$ for all 3 groups. The $260 / 230$ ratios were less than the anticipated 2.0 but were not statistically different. Previous publications have reproducibly shown that adipose-derived MSCs express markers typically used to characterize human MSCs including CD44 and CD90 [20, 21]. Thus, we focused on biomarkers of multipotency including GATA4, GATA6, SOX2 and SOX17. Biomarkers 
of multipotent cells as well as early differentiation markers were assessed with RT-qPCR. We have included both the raw $\mathrm{Ct}$ values (Table 3) and the data normalized to the housekeeping gene (Figure 4A).

Gata4 is thought to regulate embryogenesis in the mesoderm and endoderm and is upregulated with differentiation but found in low levels in undifferentiated bone marrow MSCs [22]. Gata4 expression was low in all 3 samples (>36 cycles, Table 3 ). When the data was normalized to the housekeeping gene, Gapdh, the results continued to show low expression of Gata4 (Figure 4A) with the cells from reproductive fat showing statistically more expression than the other 2 groups (lower $\Delta \mathrm{Ct}$ ).

GATA6 is a mesodermal marker involved in regulation of cell differentiation during development [23]. As expected, Gata6 expression was high in the feline cells, as indicated by the earlier cycle threshold values (Table 3). The digestion method did not seem to affect Gata6 expression as the enzymatic and the mechanical digestion performed similarly. Normalization to Gapdh expression continued to illustrate the strong expression level with no statistical difference between the 3 groups (Figure 4A).

Sox 2 is a transcription factor that is essential for pluripotency maintenance in stem cells [24] and was detected at high levels in all 3 samples. Sox2 expression was greatest in the cells digested enzymatically, showing lesser amplification in the mechanically digested samples (Table 3). Normalization to Gapdh showed the greatest expression in the subcutaneous fat that was enzymatically digested (Figure 4A).

The endodermal marker SOX17 is highly involved in the regulation of embryonic development [25]. Expression of Sox17 followed the same trend as Sox2 expression, 
demonstrating an increase in expression in the enzymatically digested tissue, whether it was from the subcutaneous or reproductive depots (Table 3). After normalization, the subcutaneous fat with enzymatic digestion had the highest expression level of Sox17 (Figure 4A).

PDX1 is a marker of early human pancreatic development as is typically only detected in late endoderm differentiation [26]. After failing to detect significant levels of Gata4 in the feline adipose tissue, we added the analysis of $P d x 1$ as a negative control. As expected, $P d x 1$ was detected only after extremely high cycle numbers (Table 3), and normalization to Gapdh did not change the results (Figure $4 \mathrm{~A}$ ).

Because both Gata4 and $P d x 1$ were consistently detected but at high cycle levels (>35 cycles), the fragment sizes were evaluated to ensure that they were the correct size and not primer dimers or contaminants. All were consistent with the expected size. Figure 4B provides examples of the amplicons showing the anticipated size for all of the biomarkers with no additional bands present. The gels were cropped to provide a standard order for the groups. The full gels are provided in the supplemental section of this manuscript (Figure S1).

\section{Cell Culture Expansion}

Cells were seeded at an approximate density of 5,000 cells $/ \mathrm{cm}^{2}$ and cell expansion measured by population doubling times. Figure 5A summarizes the differences in expansion capability for the three groups. During P1 (first passage), there was no statistically significant difference between the group doubling time, although there was a trend towards a faster doubling time for the subcutaneous tissue that was enzymatically digested. Viability of the cells 
was measured at the end of the P1 passage. The cells in these groups showed high viability with no statistical difference between groups (Figure 5B).

During passage 2 (P2), the cells from mechanically digested subcutaneous tissue maintained approximately the same doubling time as during the P1 passage, while the other 2 groups showed dramatically shorter doubling times. The reproductive group's doubling time was statistically different from the mechanically digested group (Figure $\mathbf{5 C}$ ). Viability of the cells at the end of P2 was slightly lower than the P1 cells but they were not statistically different. Within the P2 groups, the viability was not statistically different. Doubling time for P3 was similar to P1 for the reproductive group and quicker for the other 2 groups (Table 4). Viability remained high for the reproductive group at P3, but significantly lower for the 2 subcutaneous groups. Viability at P4 still averaged $92 \%$ for the reproductive group, but too few of the subcutaneous groups were passaged to $\mathrm{P} 4$ to be able to conduct statistical comparisons.

\section{DISCUSSION}

MSCs are becoming increasingly relevant to clinical care [7] but the outcomes associated with MSC treatment have shown great inconsistency. Some of the outcome variability may be due to the altered characteristics associated with the cells, based on the site of isolation of the initial tissue $[13,27]$. Other factors responsible for inducing variability may include the methods of isolation, the amount of tissue harvested, and the passage number. In felines, there have been few studies to compare sources of MSCs and they have focused on comparing bone marrow- 
derived MSC to adipose MSCs [19]. Further, when working with abdominal adipose tissue, the exact donor site often is not clarified in the reports $[28,29]$.

Obviously, a much greater quantity of adipose tissue can be harvested from the subcutaneous fat depot as compared to the reproductive depot. However, the density of MSCs obtained from those regions was vastly different with the tissue surrounding the reproductive organs resulting in 40 times more MSCs per tissue weight. Even with this great difference in yield (cells per tissue weight), the overall number of cells obtained was greater with the subcutaneous harvest due to the large volume of subcutaneous fat that could be retrieved. However, subcutaneous cells had an extremely low viability, especially when digested mechanically. These samples also had the slowest P2 doubling time. The range of doubling times reported here are consistent with some previous publications of feline abdominal adipose tissue [28-30] but slower than those reported by others $[20,31]$.

Only one other group has focused on different fat depots in felines comparing abdominal from subcutaneous locations [28]. They found that there was no difference between the two locations in the multipotent markers nor in the ability of the cells to differentiate into mesoderm lineages. However, they did not identify the exact location of the abdominal fat depot, thus it is difficult to directly compare their results to those reported here.

There have been reports suggesting that mechanical digestion can be advantageous to enzymatic digestion when small quantities of MSCs are desired due to its decreased cost and time commitment but with a decreased yield [32]. We confirmed that the yield for the mechanically digested sample was significantly less than the other 2 groups and the resulting viability of the cells was poor (approximately $25 \%$ ). While the remaining cells could be rescued 
and passaged, the poor initial outcome indicates that a significantly larger amount of starting material would be necessary to harvest if using mechanical digestion.

The quality and amount of RNA extracted from the 3 groups showed no relevant differences. This is important because of studies concluding that fat contains a high fatty acid content that can interfere with the extraction of pure RNA, indicated by the 260/230 ratio [33]. Because extensive work has already been published on the general MSC markers found in feline adipose tissue from numerous sources [19, 28, 30, 31], we opted to study biomarkers of pluripotency and lineage assignment to further characterize the tissue. While the expression levels of some of the pluripotent biomarkers reached statistical significance, the differences were small and likely not biologically relevant. Importantly, all of the biomarkers were detected at high CT values, indicating low levels of gene expression. The only exception was the housekeeping gene, Gapdh, which was detected at an average of 21 cycles.

SOX2 is a master stem cell transcription factor, determining the stemness capacity of cells [24]. High levels of expression of SOX2 have been reported in human, monkey and canine abdominal fat previously [34, 35]. In addition, cat embryos, along with undifferentiated feline neural stem cells, contain high levels of Sox2 [36-38]. In comparison with canine cells, feline amniotic MSCs showed a much lower expression of Sox2 and less immunostaining of the protein compared to canine amniotic stem cells [39]. In addition, a number of labs have shown that induced pluripotent stem cells derived from feline fibroblasts express Sox2 [40, 41]. However, the presence of Sox2 in feline adipose-derived MSCs is controversial. Some have reported the inability to detect Sox2 or the resulting protein in feline abdominal or subcutaneous adipose MSCs $[28,29]$. In contrast, our results are consistent with those of Lee et al, showing detection 
of Sox2 in the abdominal adipose tissue [31]. Although not very likely, we point out that our choice of PCR primers for Sox2 might also result in some differences compared to earlier studies. Unfortunately, ours is the only study to include the $\mathrm{Ct}$ values of our results rather than relative values that provide no indication of the absolute levels.

GATA6 encodes a transcription factor normally found in MSCs essential to the selfrenewal properties of pluripotent cells. Knockdown of GATA6 results in suppression of the selfrenewal capacity of MSCs [42]. In cats, Gata6 is assumed to be involved in differentiation similar to humans, but that has yet to be examined. Studies have described the presence of Gata6 in feline induced pluripotent stem cells [41] and in cat embryos [36], but ours is the first study to report the presence of Gata6 in feline adipose MSCs.

SOX17, identified in human adipose derived MSCs [43], is involved in the capacity of the cells to differentiate into endoderm and eventually to hepatocytes [44] or pancreatic tissue [33]. Like Gata6, Sox17 has been detected in feline induced pluripotent stem cells [41], but has not been investigated in feline adipose MSCs. Thus, this is the first study to identify Sox17 in feline adipose-derived MSCs.

GATA4 is found in high levels in human ovaries where it is associated with sex determination along with ovary growth and function, but not in adipocytes [45]. GATA4 is involved in differentiation into osteoblasts and development of the heart and has been reported to be expressed in only $15 \%$ of rat bone marrow-derived MSCs based on immunohistochemistry [22]. Thus, it was not surprising that little was detected in the adipose MSCs. In fact, minimal expression confirmed that ovarian tissue did not contaminate the reproductive fat group. 
Likewise, PDX1 is a biomarker of pancreatic development and is farther down the differentiation lineage. We included $P d x 1$ detection as a negative control, thus the lack of $P d x 1$ was expected.

While cells from all three groups studied here had similar pluripotency profiles, the improved yield (cells/tissue weight) and initial cell viability was dramatically better for the cells harvested from the reproductive tissue, which can be harvested ethically during spaying procedures. If the goal is an autologous product, then reproductive fat harvests would not be appropriate, but the field is quickly moving toward the allogeneic model. Consequently, fat surrounding the reproductive organs may offer a consistently high-quality source of MSCs. In addition, we showed that enzymatic digestion was the best approach for subcutaneous tissue and the only option available when working with reproductive tissue due to the small amount of starting material.

\section{CONCLUSION}

In conclusion, the work summarized here demonstrates that feline reproductive adipose tissue is a reasonable source of MSCs to be cultured for eventual therapeutic application. The MSCs should be isolated via predominantly enzymatic digestion, but further research should be done to continue optimizing a digestion protocol. For example, in certain clinical situations, longterm cell culture may not be an option and mechanically-digested subcutaneous fat may be the only option. In addition, further research should continue to further characterize feline adiposederived MSC, including the differentiation potentials. 


\section{METHODS}

\section{Adipose Tissue Collection}

The adipose tissue was collected from 32 healthy female cats. One set of cats were undergoing spaying at local animal shelters and the discarded tissue removed during spaying (the ovaries and uterine horn) was collected. Subcutaneous fat was removed from male and female research animals undergoing euthanasia (sodium pentobarbital) at the completion of an unrelated study. The University of Kansas Medical Center's IACUC determined that tissue removed during spaying or after euthanasia was exempt from required protocol approval. As all tissue collected would otherwise have been discarded, no consent was necessary. The licensed veterinarians overseeing the animals approved the collection of the discarded tissue. No exclusion criteria were set for the study. Table 1 summarizes the donor characteristics. All donors were standard long or shorthair cats and were fully immunized at the time of tissue retrieval. Due to the fact that tissue was retrieved during other procedures, the site of tissue collection could not be randomized. Sample sizes were based on convenience of the surgical procedures completed through the duration of the study. Due to large differences in the tissue mass obtained from the different sites, blinding of the initial sample groups could not be done. Analysis of the later passages was completed in a blinded fashion.

Reproductive adipose tissue was collected aseptically during standard spaying of healthy cats. The removed ovaries and uterine horn were placed in chilled transport medium comprised of DMEM/F12 (ThermoFisher, Cat\#124000024) containing 1\% Penicillin-Streptomycin (ThermoFisher, Cat\#15140122) and transported to the lab on ice. Under aseptic conditions, the 
fat was manually dissected from the reproductive organs. Subcutaneous adipose tissue was collected by manual excision and placed in the chilled transport medium. Immediately following dissection, the digestion protocol was initiated using either of the methods described below.

\section{Digestion of Adipose Tissue}

Mechanical Digestion

Under aseptic conditions, the tissue was rinsed, weighed, and minced using sterile scissors. Mechanical digestion involved passing the adipose tissue between two $60 \mathrm{~mL}$ syringes connected with an anaerobic luer-to-luer connector (Tulip, Cat \#TP ATLLLL2.4). Using the $2.4 \mathrm{~mm}$ Tulip connector, the tissue was passed between the two syringes 10 times and then passed through the smaller $1.2 \mathrm{~mm}$ Tulip connector (Tulip, Cat\#TP ATLLLL1.2) another 10 times. The tissue solution was centrifuged at $550 x g$ for 10 minutes. The cell pellet was reserved for plating in DMEM culture medium. The remaining supernatant was collected and the digestion was repeated twice more with increasing centrifugation speeds in each round of digestion (620xg and 690xg, respectively). The cells from the pellets from each digestion were collected and counted. The pellets were resuspended in DMEM:F12 with 10\% FBS (ThermoFisher, Cat \#26140087) and $1 \%$ Penicillin-Streptomycin and cultured at $37^{\circ} \mathrm{C}, 5 \% \mathrm{CO}_{2}$ in a humidified chamber.

\section{Enzymatic Digestion}

Under aseptic conditions, the tissue was rinsed, weighed, and minced using sterile scissors and digested in a $0.6 \mathrm{WU} / \mathrm{mL}$ Liberase (Roch, Liberase MNP-S, /cat \#06297790001) for 30 minutes at $37^{\circ} \mathrm{C}$. For the larger tissue amounts of subcutaneous fat, the tissue was passed 
through a $100 \mathrm{~mm}$ filter and centrifuges at $260 \times \mathrm{xg}$ for $5 \mathrm{~min}$. The supernatant was removed and mixed with $5 \mathrm{~mL}$ of red blood cell lysis buffer (Millipore Sigma, Cat \#11814389001) followed by centrifugation at $260 \times \mathrm{xg}$ for $5 \mathrm{~min}$. The final supernatant was resuspended in DMEM:F12 with 10\% FBS and $1 \%$ Penicillin-Streptomycin and cultured at $37^{\circ} \mathrm{C}, 5 \% \mathrm{CO}_{2}$ in a humidified chamber.

\section{Cell Culture}

Following digestion, cells were incubated for 48 hours at $37^{\circ} \mathrm{C}$ and $5 \% \mathrm{CO}_{2}$ after which the non-adherent cells were removed, and fresh medium was added. The remaining plasticadherent cells were trypsinized (Worthington Cat\# 9002-07-7) and re-plated at an average density of 5,000 cells $/ \mathrm{cm}^{2}$ into T175 flasks. Cell numbers were calculated using automated cell counter (EVE; NanoEnTek) at the time of passage and during expansion in order to calculate the doubling time. Cells were passaged when they met the requirement of $80-100 \%$ confluency. Flasks that failed to obtain $80 \%$ confluency were removed from analysis. Images of cultured cells were obtained using an Axio Vert.A1 Inverted microscope (Zeiss) with a 10X objective. Cells were not cultured beyond 5 passages.

Doubling time was calculated based on a published protocol [46].

$$
\mathrm{DT}=\mathrm{T}^{*} \ln 2 / \ln (\mathrm{Xe} / \mathrm{Xb}-1)
$$

$\mathrm{T}$ is time, In is the natural log, $\mathrm{Xb}$ indicates the starting cell number, and $\mathrm{Xe}$ indicates the ending cell number.

\section{Viability}


Viability was measured from fluorescence emission levels of calcein (ThermoFisher, Cat \#C3099) and propidium iodide (ThermoFisher, Cat \#P3566) on a multi-mode plate reader (BioTek Cytation 5). Calcein stains live cell green and PI stains dead cells as red. Viability was calculated as:

[live cell emission (green)/total cells $($ green + red) $] \times 100$

Trypan blue exclusion staining was also used as a measure of viability. The cells were dispersed into single cell suspensions using trypsin and briefly incubated with Trypan blue. Viability was determined as the ratio of (live cells / total number of cells) $\times 100$.

\section{Biomarker Expression}

Total RNA was extracted from frozen cell pellets of the digested adipose tissue using the mirVana RNA Extraction kit (ThermoFisher, Cat\#AM1560) per the manufacturer's protocol. RNA quality was assessed using the NanoDrop 1000 Spectrophotometer (Thermo-Fisher). One $\mu \mathrm{g}$ of total RNA was used to make cDNA using SuperScript IV Reverse Transcriptase (Invitrogen, Cat $\# 18090010)$ as per the manufacturer's protocol using oligo (dT). Primer sequences for the biomarkers were determined using BLAST (Table 2) and confirmed via gel electrophoresis.

The cDNA was run through RT-qPCR on the Applied Biosystems ViiA7 PCR System using Power SYBR Green PCR Master Mix (Applied Biosystems, Cat \#4367659) using the following protocol: 2 minutes at $95^{\circ} \mathrm{C}$ for initial denaturation, followed by cycling 15 seconds at $95^{\circ} \mathrm{C}$ for denaturation, 10 seconds at $60^{\circ} \mathrm{C}$ for annealing, and 60 seconds at $70^{\circ} \mathrm{C}$ for extension. This protocol repeated for 49 cycles, followed by recording a melt curve. All samples were amplified in triplicate. An automatic threshold was utilized and a cut off of 40 cycles was determined to 
indicate presence or absence of biomarkers. Negative controls without template were run to 50 cycles with no amplification. The cycle threshold values were normalized to Gapdh.

\section{Statistical Analysis}

Unpaired T-tests were conducted on the demographic data (Table 1). All remaining data were analyzed using a one-way analysis of variance (ANOVA) on ranks with post hoc Dunn's pairwise comparison was performed using SigmaPlot 13.0. $P$ values of less than 0.05 were considered statistically significant, noted by symbols in the graphs. Data are presented as averages \pm SEM. 


\section{FIGURE LEGENDS}

Figure 1: Density of isolated MSCs from adipose tissue.

Following adhesion to plastic, the MCSs were counted and the value divided by the initial weight of the tissue for that sample. The adipose tissue surrounding the reproductive organs had the highest density of cells per gram of tissue. ${ }^{*}$ indicates statistical differences $(p<0.05)$ compared to the reproductive enzymatically-digested group.

\section{Figure 2: Initial viability of the isolated MSCs.}

MSCs isolated from reproductive adipose tissue demonstrated significantly more viable cells when compared to MSCs isolated from subcutaneous adipose tissue digested either enzymatically or mechanically. ${ }^{*}$ indicates statistical differences $(p<0.05)$ compared to the reproductive enzymatically-digested group.

\section{Figure 3: MSC morphology.}

Following digestion, plated cells that adhered to plastic were cultured to confluency. There were no obvious differences between the groups, although cells from the reproductive group had the most consistent appearance. Scale bar $=200 \mu \mathrm{m}$.

\section{Figure 4: Biomarker Expression.}

A) Five biomarkers for pluripotency, differentiation and expansion were measured via rtPCR. The $\triangle C T$ values, normalized to the housekeeping gene Gapdh, showed relatively high 
expression of pluripotent and expansion markers, Gata6, Sox2 and Sox17. * indicates a statistically significant difference between the reproductive tissue compared to the other two groups. \# indicates statistically significant differences between the subcutaneous enzymatically-digested fat and the other two groups. B) Examples of amplicons with single bands of the anticipated sizes are shown. RE = Reproductive Enzymatic Group, SE = Subcutaneous Enzymatic Group, SM = Subcutaneous Mechanical Group.

\section{Figure 5: Cell Expansion}

A) The doubling time for the 3 groups at P1 shows high variability and no statistical differences. B) Viability of those same cells was nearly identical and very high. C) P2 doubling time showed less variability with the slowest doubling from the mechanically-digested subcutaneous tissue ( $p, 0.05)$. D) The viability of the P2 cells was lower than P1, but not statistically different between the passages or within the P2 passage. (* $P<0.05)$.

\section{Supplemental Figure 1: Figure 4B Original Gels}

The gels shown in Figure 4B were cropped from the original gels shown here, which included amplicons for this study as well as another study comparing different types of serum, indicated as "Other Study" in the figure. 


\section{ABBREVIATIONS}

$\mathrm{CO}_{2} ;$ Carbon dioxide

$\mathrm{cm}^{2}$; centimeter squared

CD; Cluster of differentiation

$\mathrm{Ct}$; threshold cycle

$\Delta \mathrm{Ct}$; Change in $\mathrm{Ct}$

dT; deoxythymine

DT; doubling time

DMEM/F12; Dulbecco's Modified Eagle Medium F-12

g; grams

$\mathrm{mm}$ millimeter

MSC; multipotent stromal cells

$\mathrm{P} 1, \mathrm{P} 2$ or $\mathrm{P} 3$; passage number

$\mathrm{RE}$; reproductive enzymatic

RT-qPCR; Quantitative reverse transcription polymerase chain reaction

RNA; ribonucleic acid

SM; subcutaneous mechanical

SE; subcutaneous enzymatic

T; time

WU; Wunsch units

$\mathrm{Xb}$; Starting cell number

Xe; Ending cell number 
$\mathrm{Xg}$; times gravity

\section{DECLARATIONS}

- Ethics approval and consent to participate: The University of Kansas Medical Center's IACUC determined that tissue removed during spaying or after euthanasia was exempt from required protocol approval.

- Consent for publication: Not applicable

- Availability of data and materials: Data generated and analyzed for the current study are available from the corresponding author.

- Competing Interests: $\quad$ LSB is part owner of Likarda, LLC.

- Funding: $\quad$ Financial support for the fellowship of Dr. P. Ortiz was provided by the Young Leaders of the Americas Initiative. Research funding was supplied by the Kansas City University Student Research Fund. The funding bodies played no role in the design, analysis or reporting of the study.

- Authors contribution: AW completed all PCR work, assisted with analysis of other data and wrote the manuscript. PO designed the study and assisted in tissue collection and cell culture, DB is the academic mentor to AW and oversaw her work. LO conducted all cell culture and the associated assays including imaging, FK oversaw all initial digestions 
and designed the biomarker component to the study. MF assisted AW with biomarker analysis, LSB oversaw the experiments, analysis and manuscript writing.

- Acknowledgements: The authors wish to acknowledge S. Janette Williams and Dr. Nataliya Kibiryeva for support throughout the project.

- Authors' Information (optional): Not applicable. 


\section{REFERENCES}

1. Zomer H, Vidane A, Goncalves N, Ambrosio C. Mesenchymal and induced pluripotent stem cells: general insights and clinical perspectives. Stem Cells Cloning. 2015; 8:125134.

2. Luo L, Hu D, Yin J, Xu R. Molecular Mechanisms of Transdifferentiation of AdiposeDerived Stem Cells into Neural Cells: Current Status and Perspectives. Stem Cells Int. 2018; 2018:5630802.

3. Golpanian S, Wolf A, Hatzistergos K, Hare J. Rebuilding the Damaged Heart: Mesenchymal Stem Cells, Cell-Based Therapy, and Engineered Heart Tissue. Physiol Rev. 2016; 96:1127-1168.

4. Wang J, Chen Z, Sun M, Xu H, Gao Y, Liu J, Li M. Characterization and therapeutic applications of mesenchymal stem cells for regenerative medicine. Tissue and Cell. 2020; 64:101330.

5. Peng Y, Ke M, Xu L, Liu L, Chen X, Xia W, Li X, Chen Z, Ma J, Liao D et al. Donor-derived mesenchymal stem cells combined with low-dose tacrolimus prevent acute rejection after renal transplantation: a clinical pilot study. Transplantation. 2013; 85(1):161-168.

6. Regmi S, Pathak S, Kim J, Yong C, Jeong J. Mesenchymal stem cell therapy for the treatment of inflammatory diseases: Challenges, opportunities, and future perspectives. Eur J Cell Biol. 2019; 98:151041.

7. Marx C, Silveira M, Nardi N. Adipose-derived stem cells in veterinary medicine: characterization and therapeutic applications. Stem Cells Devel. 2015; 24(7):803-813. 
8. Trzil J, Masseau I, Webb T, Chang C-H, Dodam J, Liu H, Quimby J, Dow S, Reinero C. Intravenous adipose-derived mesenchymal stem cell therapy for the treatment of feline asthma: a pilot study. J Feline Med Surg. 2016; 18(12):981-990.

9. Quimby J, Borjesson D. Mesenchymal stem cell therapy in cats: current knowledge and future potential. J Feline Med Surg. 2018, 20:208-216.

10. Thomson A, Berent A, Weisee C, Langston C. Intra-arterial renal infusion of autologous mesenchymal stem cells for treatment of chronic kidney disease in cats: phase I clinical trial. J Vet Int Med. 2019; 33(3):1351-1361.

11. Sato K, Yamawaki-Ogata A, Kanemoto I, Usui A, Narita Y. Isolation and characterization of peripheral blood-derived feline mesenchymal stem cells. Vet J. 2016; 216:183-188.

12. Zazzeroni L, Lanzoni G, Pasquinelli G, Ricordi C. Considerations on the harvesting site and donor derivation for mesenchymal stem cells-based strategies for diabetes. CellR4 Repair Replace Regen Reprogram. 2017; 5(5):e2435.

13. Lavrentieva A, Hoffmann A, Lee-Thedieck C. Limited potential or unfavorable manipulations? Strategies toward efficient mesenchymal stem/stromal cell applications. Front Cell Dev Biol. 2020; 8:316.

14. Coelho M, Oliveira T, Fernandes R. Biochemistry of adipose tissue: an endocrine organ. Arch of Med Sci. 2013; 9:191-200.

15. Ibrahim M. Subcutaneous and visceral adipose tissue: structural and functional differences. Obes Rev. 2010; 11:11-18. 
16. Guercio A, Di Bella S, Casella S, Di Marco P, Russo C, Piccione G. Canine mesenchymal stem cells (MSCs): characterization in relation to donor age and adipose-tissueharvesting site. Cell Biol Int. 2013; 37(8):789-798.

17. Ritter A, Friemel A, Roth S, Kreis N, Hoock S, Safdar B, Fischer K, Mollmann C, Solbach C, Louwen F et al. Subcutaneous and Visceral Adipose-Derived Mesenchymal Stem Cells: Commonality and Diversity. Cells. 2019; 8(10):1288.

18. Voga M, Adamic N, Vengust M, Majdic G. Stem Cells in Veterinary Medicine - Current State and Treatment Options. Front Vet Sci .2020; 7:278.

19. Webb T, Quimby J, Dow S. In vitro comparison of feline bone marrow-derived and adipose tissue-derived mesenchymal stem cells. J Feline Med Surg. 2012; 14:165-168.

20. Panasophonkul S, Samart P, Kongon K, Sathanawongs A. Phenotypic characteristics of feline adipose-derived stem cells affected by cell passage number. Pol J Vet Sci. 2017; 20(4):651-660.

21. Kono S, Kazama T, Kano K, Harada K, Uechi M, Matsumoto T. Phenotypic and functional properties of feline dedifferentiated fat cells and adipose-derived stem cells. Vet J. $2014 ; 199(1): 88-96$.

22. Mangi A, Noiseux N, Kong D, He H, Rezvani M, Ingwall J, Dzau V. Mesenchymal stem cells modified with Akt prevent remodeling and restore performance of infarcted hearts. Nat Med. 2003; 9(9):1195-1201.

23. Sagi B, Marahgechi P, Urban V, Hegyi B, Szigeti A, Fajka-Boja R, Kudlik G, Nemet K, Monostori E, Gocza E et al. Positional identity of murine mesenchymal stem cells 
resident in different organs is determined in the postsegmentation mesoderm. Stem Cells Devel. 2012; 21(5):814-828.

24. Kozlowska U, Krawczenko A, Futoma K, Jurek T, Rorat M, Patrzalek D, Klimczak A.. Similarities and differences between mesenchymal stem/progenitor cells derived from various human tissues. World J Stem Cells. 2019; 11(6):347-374.

25. Kanai-Azuma M, Kanai Y, Gad J, Tajima Y, Taya C, Kurohmaru M, Sanai Y, Yonekawa H, Yazaki K, Tam P et al. Depletion of definitive gut endoderm in Sox17-null mutant mice. Development. 2002; 129(10):2367-2379.

26. Zhu Y, Liu Q, Zhou Z, Ikeda Y. PDX1, Neurogenin-3, MAFA: critical transcription regulators for beta cell development and regeneration. Stem Cell Res Ther. 2017; 8:240.

27. Wada Y, Ikemoto T, Morine Y, Imura S, Saito Y, S Y, Shimada M. The Differences in the Characteristics of Insulin-producing Cells Using Human Adipose-tissue Derived Mesenchymal Stem Cells from Subcutaneous and Visceral Tissues. Sci Rep. 2019; 9:13204.

28. Gomez M, Qin Q, Biancardi M, Galiguis J, Dumas C, MacLean R, Wang G, Pope C. Characterization and multilineage differentiation of domestic and black-footed cat mesenchymal stromal/stem cells from abdominal and subcutaneous adipose tissue. Cell Reprogam. 2015; 17(5):376-392.

29. Kim H-R, Lee J, Byeon J, Gu N-Y, Lee J, Cho I-S, Cha S-H. Extensive characterization of feline intra-abdominal adipose-derived mesenchymal stem cells. J Vet Sci. 2017; 18(3):299-306. 
30. Clark K, Fierro F, Ko E, Walker N, Arzi B, Tepper C, Dahlenburg H, Cicchetto A, Kol A, Marsh L et al. Human and feline adipose-derived mesenchymal stem cells have comparable phenotype, immunomodulatory functions, and transcriptome. Stem Cell Res Ther. 2017; 8:69.

31. Lee B-Y, Li Q, Song W-J, Chae H-K, Kweon K, Ahn J-O, Youn H-Y. Altered properties of feline adipose-derived mesenchymal stem cells during continuous in vitro cultivation. J Vet Med Sci. 2018; 80(6):930-938.

32. Aronowitz J, Lockhart R, Hakakian C. Mechanical versus enzymatic isolation of stromal vascular fraction cells from adipose tissue. Springerplus. 2015; 4:713.

33. Spence J, Lange A, Lin S, Kaestner K, Lowy A, Kim I, Whitsett J, Wells J. Sox17 regulates organ lineage segregation of ventral foregut progenitor cells. Dev Cell. 2009; 17(1):6274.

34. Izadpanah R, Trygg C, Patel B, Kriedt C, Dufour J, Gimble J, Bunnell B. Biologic properties of mesenchymal stem cells derived from bone marrow and adipose tissue. J Cell Biochem. 2006; 99:1286-1297.

35. Takemitsu H, Zhao D, Yamamoto I, Harada Y, Michishita M, Arai T. Comparison of bone marrow and adipose tissue-derived canine mesenchymal stem cells. BMC Vet Res. 2012; 8:150.

36. Filliers M, Goossens K, VanSoom A, Merlo B, Pope C, De Rooster H, Smits K, Vandaele L, Peelman L. Gene expression profiling of pluripotency and differentiation -related markers in cat oocytes and preimplantation embryos. Reprod Fertil Dev. 2012; 24(5):691-703. 
37. Alexanian A, Crowe M, Kurpad S. Efficient differentiation and integration of lineagerestricted neural precursors in the traumatically injured adult cat spinal cord. J Neuroscie Meth. 2006; 150:41-46.

38. Klassen H, Schwartz P, Ziaeian B, Nethercott H, Young M, Bragadottir R, Tullis G, Warfvinge K, Narfstrom K. Neural precursors isolated from the developoing cat brain show retinal integration following transplantation to the retina of the dystrophic cat. Vet Ophthalmol. 2007; 10(4):245-253.

39. Cardoso M, Pinheiro A, Vidane A, Casals J, de Oliveira V, Goncalves N, Martins D, Ambrosio C. Characterization of teratogenic potential and gene expresssion in canine and feline amniotic membrane-derived stem cells. Reprod Dom Anim. 2017; 52:58-64.

40. Dutton L, Dudhia J, Guest D. Inducing pluripotency in the domestic cat (felis catus). Stem Cells Devel. 2019; 28(19):1299-1309.

41. Zhou T, Comizzoli P, Keefer C. Endogenous pluripotent factor expression after reprogramming cat fetal fibroblasts using inducible transcription factors. Mol Reprod Dev. 2019; 86(11):1671-1681.

42. Kubo H, Shimizu M, Taya Y, Kawamoto T, Michia M, Kaneko E, Igarashi A, Nishimura M, Segoshi K, Shimazu Y et al. Identification of mesenchymal stem cell (MSC)-transcription factors by microarray and knockdown analyses and signature molecule-marked MSC in bone marrow by immunohistochemistry. Genes to Cells. 2009; 14(3):407-424.

43. Poloni A, Maurizi G, Leoni P, Serrani F, Mancini S, Frontini A, Zingaretti M, Siquini W, Sarzani R, Cintio S. Human dedifferentiated adipocytes show similar properties to bone 
marrow-derived mesenchymal stem cells. Tissue-Specific Stem Cells. 2012; 30(5):965-

974.

44. Buyl K, Vanhaecke T, Desmae T, Lagneaux L, Rogiers V, Najar M, de Kock J. Evaluation of a new standardized enzymatic isolation protocol for human umbilical cord-derived stem cells. Toxicol in Vitro. 2015; 29(6):1254-1262.

45. Tremblay M, Sanchez-Ferras O, Bouchard M. GATA transcription factors in development and disease. Development. 2018; 145(dev164384).

46. Korzynska A, Zychowica M. A method of estimation of the cell doubling time on basis of the cell culture monitoring data. Biocybernetics Biomed Eng. 2008; 28(4):75-82. 


\section{Figures}

Figure 1

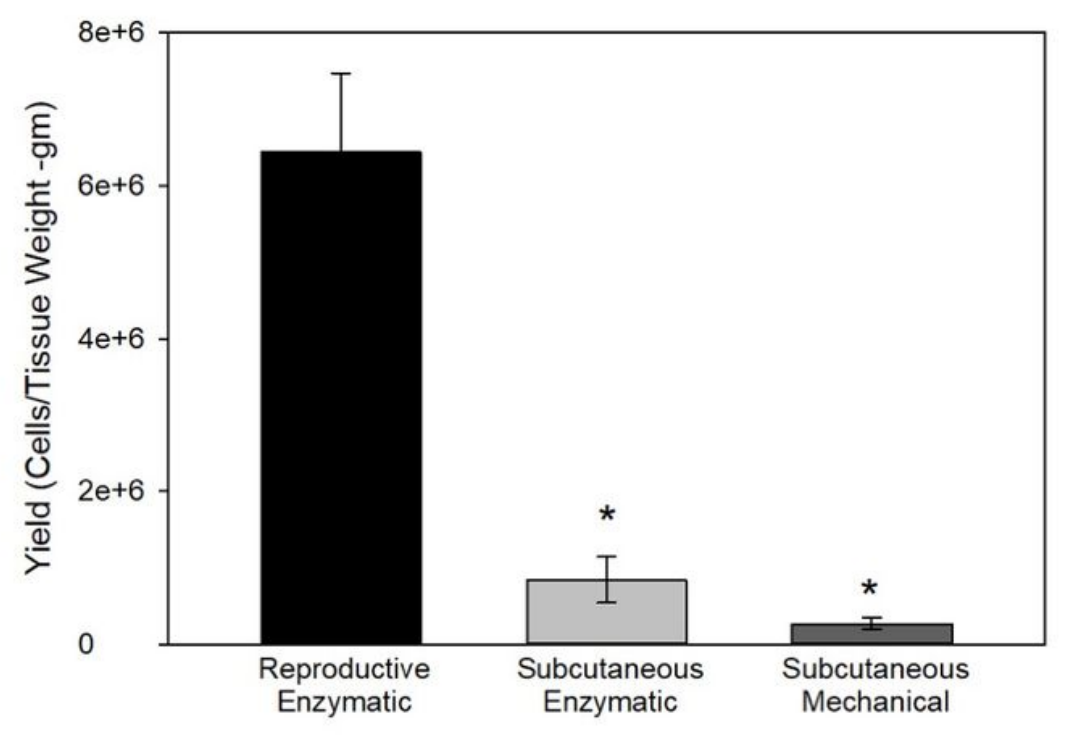

\section{Figure 1}

Density of isolated MSCs from adipose tissue. Following adhesion to plastic, the MCSs were counted and the value divided by the initial weight of the tissue for that sample. The adipose tissue surrounding the reproductive organs had the highest density of cells per gram of tissue. * indicates statistical differences $(p<0.05)$ compared to the reproductive enzymatically-digested group. 
Figure 2

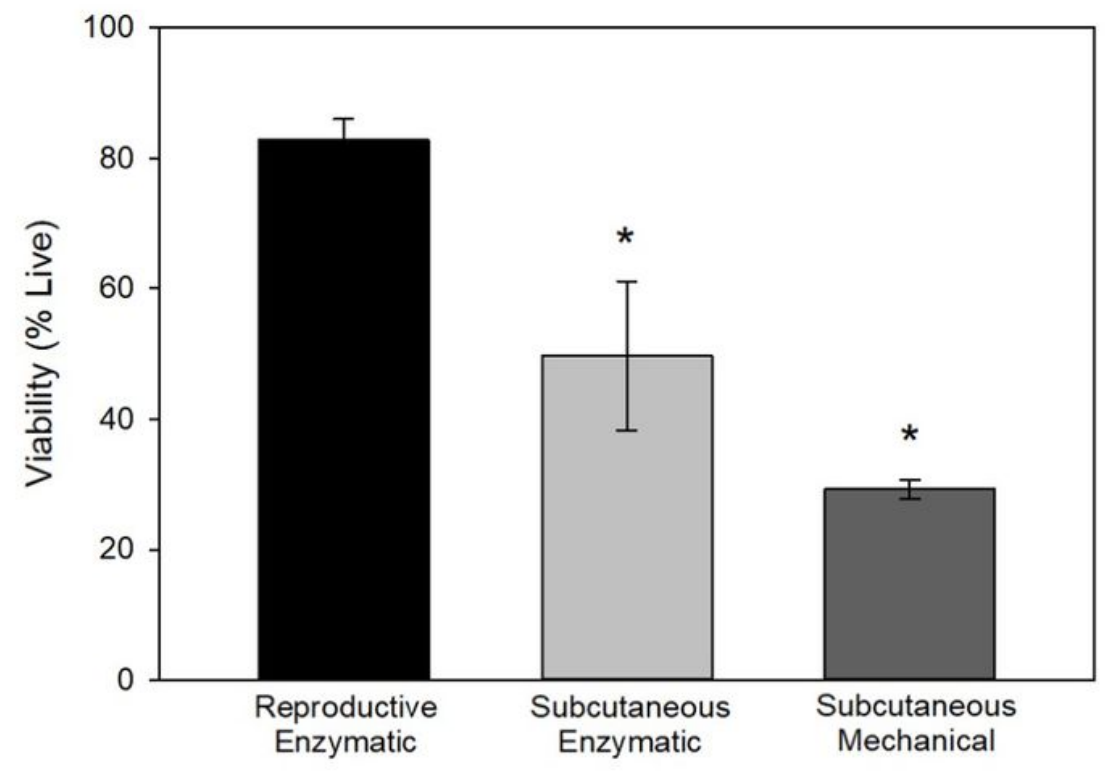

Figure 2

Initial viability of the isolated MSCs. MSCs isolated from reproductive adipose tissue demonstrated significantly more viable cells when compared to MSCs isolated from subcutaneous adipose tissue digested either enzymatically or mechanically. * indicates statistical differences $(p<0.05)$ compared to the reproductive enzymatically-digested group. 
Figure 3
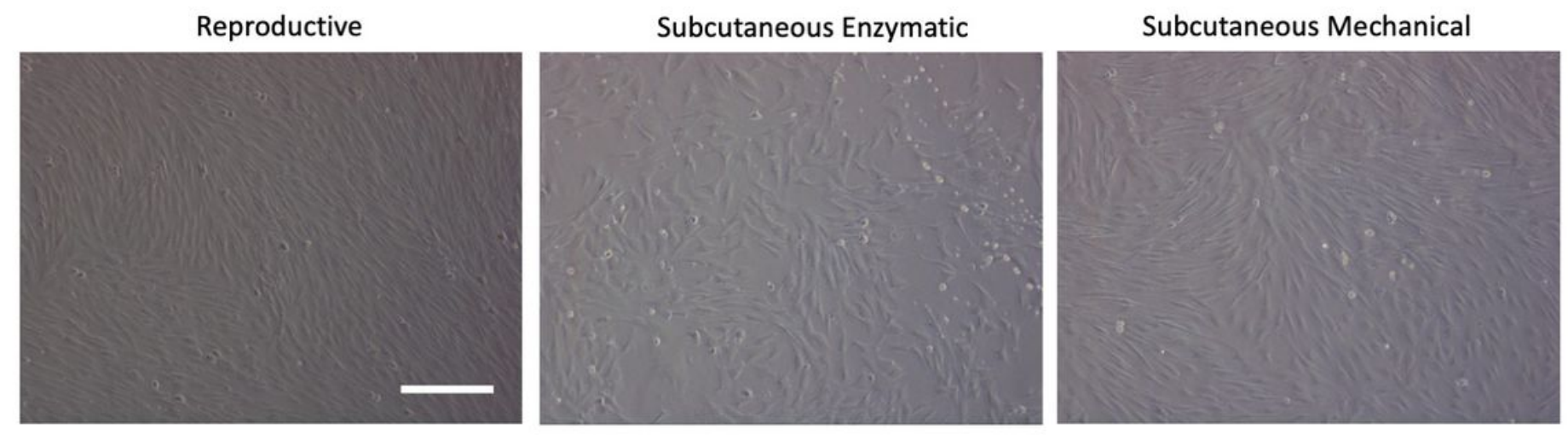

\section{Figure 3}

MSC morphology. Following digestion, plated cells that adhered to plastic were cultured to confluency. There were no obvious differences between the groups, although cells from the reproductive group had the most consistent appearance. Scale bar $=200 \mu \mathrm{m}$. 
Figure 4A

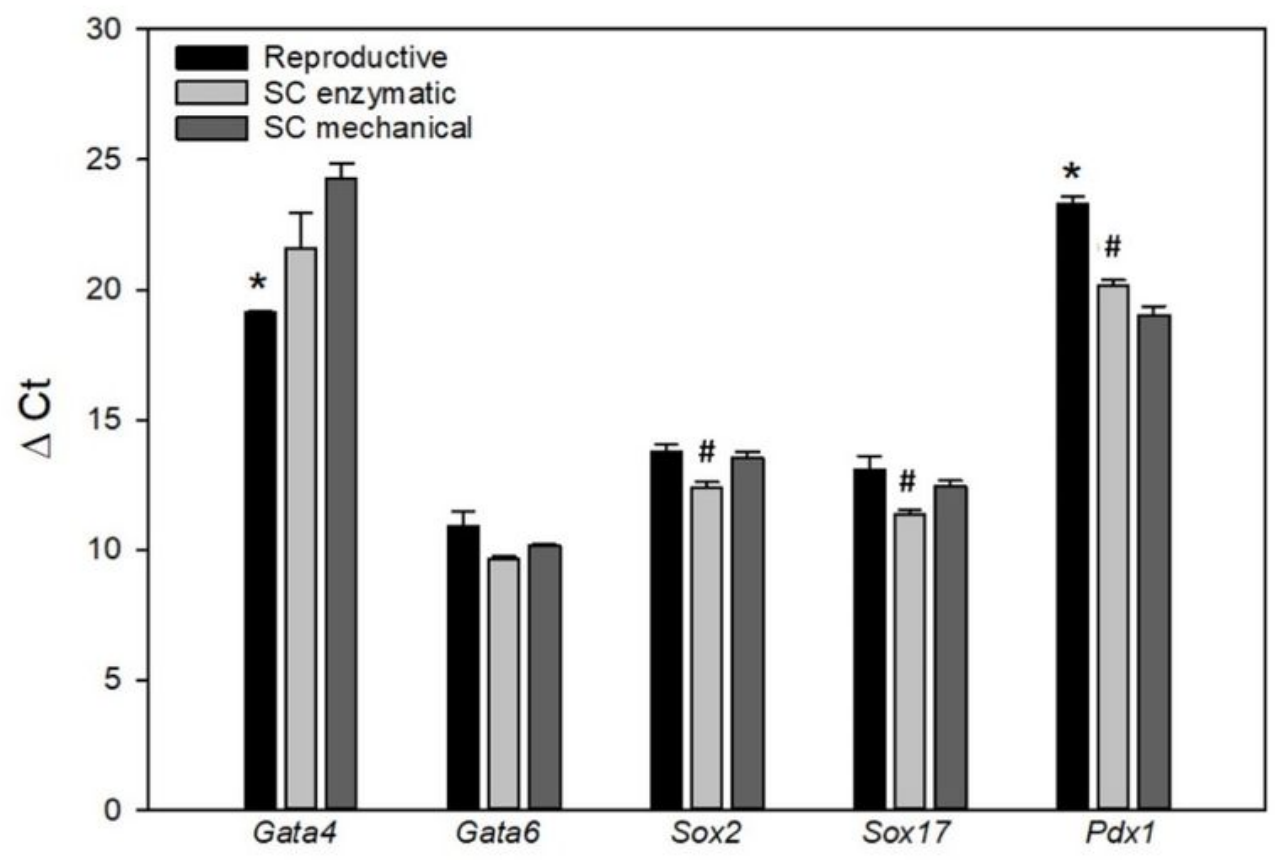

Figure 4B

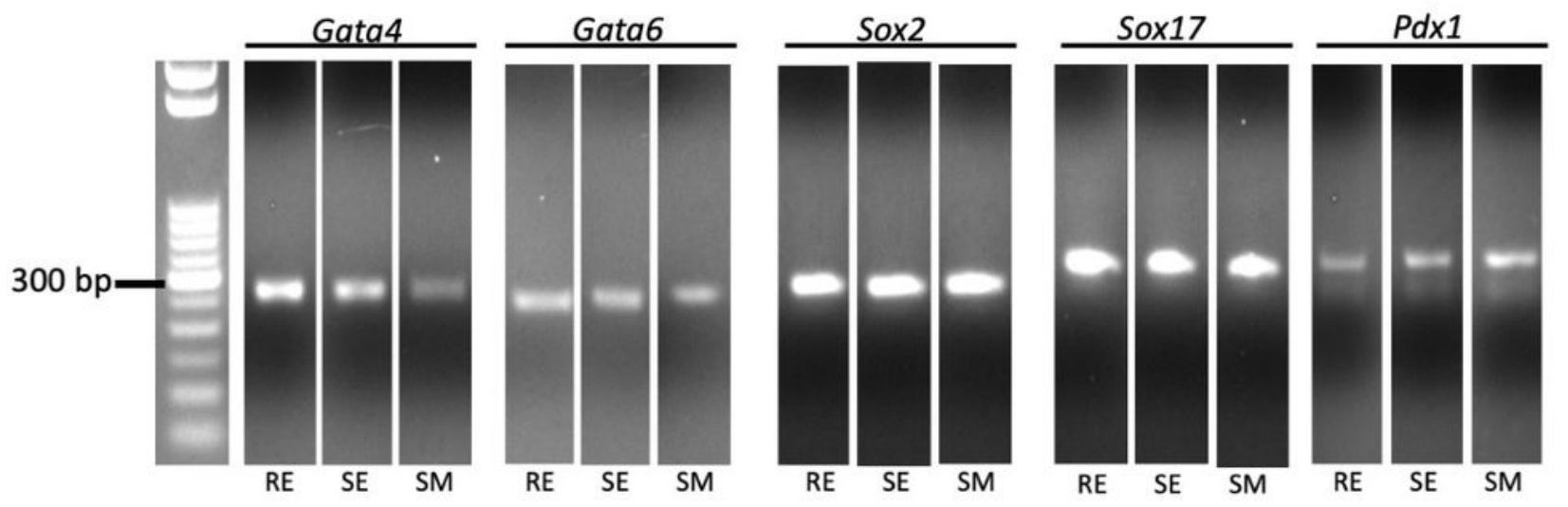

Figure 4

Biomarker Expression. A) Five biomarkers for pluripotency, differentiation and expansion were measured via itPCR. The DCT values, normalized to the housekeeping gene Gapdh, showed relatively high expression of pluripotent and expansion markers, Gata6, Sox2 and Sox17. * indicates a statistically significant difference between the reproductive tissue compared to the other two groups. \# indicates statistically significant differences between the subcutaneous enzymatically-digested fat and the other 
two groups. B) Examples of amplicons with single bands of the anticipated sizes are shown. $\mathrm{RE}=$ Reproductive Enzymatic Group, SE = Subcutaneous Enzymatic Group, SM = Subcutaneous Mechanical Group.

Figure 5
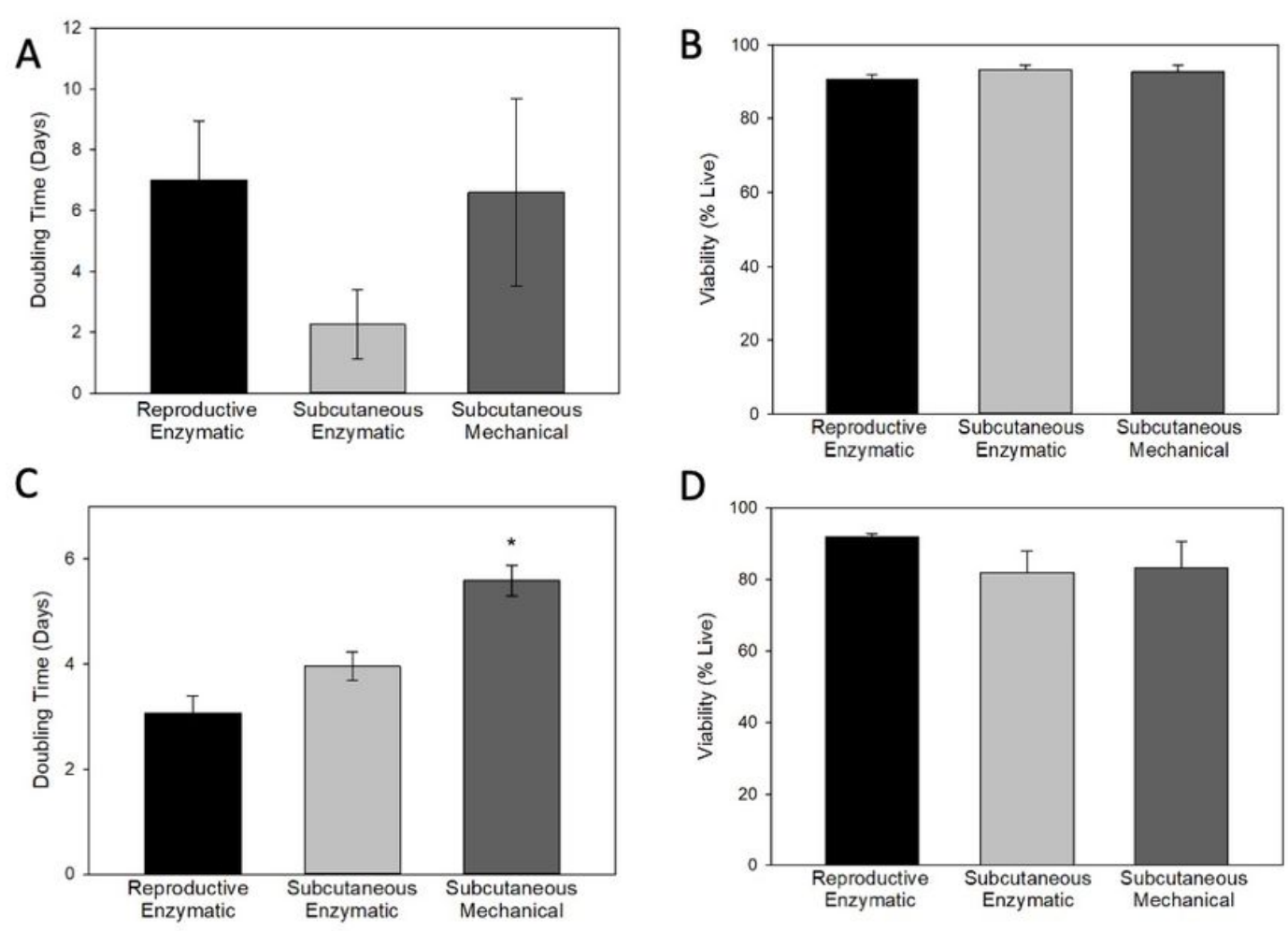

\section{Figure 5}

Cell Expansion A) The doubling time for the 3 groups at P1 shows high variability and no statistical differences. B) Viability of those same cells was nearly identical and very high. C) P2 doubling time showed less variability with the slowest doubling from the mechanically-digested subcutaneous tissue ( $p$, 0.05). D) The viability of the $P 2$ cells was lower than $P 1$, but not statistically different between the passages or within the $P 2$ passage. $\left({ }^{*} P<0.05\right)$.

\section{Supplementary Files}

This is a list of supplementary files associated with this preprint. Click to download.

- Wysong2020Tables.pdf

- SuplementalFigure1.pdf 